\title{
A Computational Study of The Effect of Blade Thickness on Performance of Vertical Axis Wind Turbine
}

\author{
M. Jahanmiri ${ }^{1}$, A. Shooshtaryrezvany ${ }^{2}$, M. Nirooei ${ }^{3}$ \\ ${ }^{1}$ Associate Professor, Dept. of Mechanical \& Aerospace Engineering, Shiraz Univ. of Tech., Shiraz, Iran \\ ${ }^{2}$ M.E.Graduate, Dept. of Mechanical \& Aerospace Engineering, Shiraz Univ. of Tech., Shiraz, Iran \\ ${ }^{3}$ M.E.Graduate, Dept. of Aerospace Eng., Sharif Univ. of Tech., Tehran, Iran,
}

\begin{abstract}
Vertical axis wind turbine with straight blade is one of the modern vertical axis wind turbines for converting wind to electrical energy. Power production capability at low speed and turbulent winds and, low disturbing noise with good appearance, makes it suitable to use small scale of this type turbine in urban areas. In view of considering importance of blade aerodynamics in turbine performance, the flow over vertical axis wind turbine with straight blade is simulated by two dimensional computational fluid dynamics. In this regard, several conventional NACA series airfoils are chosen and the effect of airfoil thickness on turbine performance was studied. Blade geometry and grid generation were created in Gambit modeling software and, Fluent software which is based on finite volume method is used for solving the problem. For Numerical solution of unsteady flow around the airfoil, the Reynolds Averaged Navier-Stokes (RANS) have been used. The simulation results and comparison of power coefficient of vertical axis wind turbine in different thickness blades showed NACA 0012 airfoil has the best coefficient of performance in a range of tip speed ratio ( $\lambda>4)$. Such values are selected for tip speed ratio, so that the dynamic stall area of the blade to be prevented.
\end{abstract}

Keywords: vertical axis wind turbine, computational fluid dynamics, unsteady flow over airfoil, oscillating airfoil

\section{Introduction}

In recent decades, generating electricity through wind turbines has been gone up rapidly all over the world. The main motivation is growing demands of reducing air pollution and issued $\mathrm{CO}_{2}$ into atmosphere and supplying increasing power demand[1]. A potential alternative for generating power in the future is installing wind turbines in urban areas [2,3]. The major advantage of using these turbines is their capability to produce electric power in places where transmitting electricity to them due to loses and cable costs is not economical[3].

Since 1970, due to the energy crisis and the surge in oil prices, a new attitude toward the use of renewable energy sources, aspecially wind power has been shaped. Industrial countries particularly USA and Canada have been investing in generating electricity by means of modern wind turbines. Sandia National Institute of America is one of the most prominent centers which has been active in developing vertical axis wind turbines in the last three decades. Engineer's investigation in this research center led to constructing a vertical axis wind turbine model "Darrieus" with a rotor diameter 24 meters in 1978. Three codes for optimizing design parameters, analyzing force and vibrations of this turbine were employed[4]. Earlier evaluating flow field around blades had been generally limited to analytical and experimental methods [5,6].

With outstanding progress in computers in the last two decades, computational fluid dynamics has been used as a tool to simulate the flow. With determined boundary and initial conditions flow equations are solved and its behavior is predicted. Recent develops in the graphics processing unit (GPU) and the central processing unit (CPU) together with utilizing three dimensional models, makes the procedure of providing computational fluid dynamics model and assessing results more convenient. In this regard some three dimensional models of horizontal axis wind turbines were introduced [7].

In the past ten years several studies were accomplished in order to optimize various parameters affecting the performance of Darrieus vertical-axis wind turbines with straight blades. Fiber-reinforced plastic (FRP) is proposed as an appropriate material for use in mid-size vertical axis wind turbine blades[8]. Islam et al. in 2007 designed a special airfoil for the small size vertical axis wind turbines with capability of generating momentum at low tip speed ratios while efficiency was favorable [9]. Islam in 2008 investigated empirically the effect of the asymmetry of airfoil on efficiency of vertical axis wind turbines. Regarding efficiency, results showed symmetric airfoils were superior to non-symmetric airfoils [10]. In the same year, Edwards et al. considered a small vertical axis wind turbine numerically and experimentally. The impact of roughness on performance was also taken into account [11].

In 2011, Wang et al. evaluated the aerodynamic performance of vertical axis wind turbine with straight blade in various speeds of wind[12]. In this year, .Habtamu and Yingxue carried out a research on self-starting of vertical axis wind turbine [13]. The impact of blade number on a straight bladed vertical-axis Darreius wind 
turbine was studied by Castelli et al. in 2012. It was found that the efficiency of this type of turbine with three blades is higher than four or five blades [14].

According to previous investigations, less attention has been paid to the blade geometry. Therefore, in present investigation the unsteady flow around the blade of vertical axis wind turbine with straight blade was simulated numerically as well as analyzing the effect of blade thickness on turbine performance.

\section{Theory of The Problem}

In Fig. 1 (a) a simple schematic of a vertical axis wind turbine with three straight blades are shown. Despite the simplicity of this type of turbine, aerodynamic analysis of flow and forces on each blade is complex. One of the complexities is change of relative velocity (W) that experiences with each blade in $360^{\circ}$ rotation and at various azimuth angles. To clarify the issue, in Fig. 1 (b) a turbine blade in an arbitrary position at azimuth angle of $\theta$ is illustrated. It is necessary to mention that azimuth angle is zero when the blade is precisely at the top of its rotational path. Counter clockwise movement increases azimuth angle in a way that reaches 360 degrees at the endpoint of rotational path.

Assuming constant wind velocity (V), direction and magnitude, over the turbine blade at every given

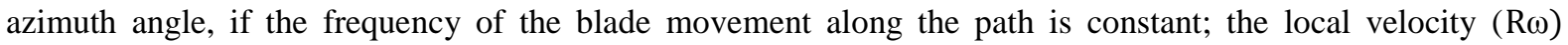
remains steady. However the direction of the above vector changes in every position of the blade. As a result, the direction and the magnitude of the relative velocity vector which is the resultant of wind velocity and the local velocity of the blade with regard to the blade azimuth angle varies and has a harmonic behavior in every revolution. Therefore, acted aerodynamic forces on the blade changes harmonically together with the variation of azimuth angle in every 360 degrees rotation.

According to the schematic drawing in the bottom of the Fig. 1 (b) equation (1) is expressed as:

$\tan \alpha=\frac{V \sin \theta}{R \omega-V \cos \theta}$ 1

One of the significant relevant parameters to wind turbines is the tip speed ratio $\lambda$. Tip speed ratio is a dimensionless parameter and is defined by equation (2).

$\lambda=\frac{\text { Tip speed of blade }}{\text { Wind speed }}=\frac{R \omega}{\mathrm{V}}$

According to expression (2), equation (1) is expressed as follows,

$\tan \alpha=\frac{\frac{V}{V} \sin \theta}{\frac{R \omega}{V}-\frac{V}{V} \cos \theta}=\frac{\sin \theta}{\lambda-\cos \theta}$

In other words.

$$
\alpha=\arctan \left(\frac{\sin \theta}{\lambda-\cos \theta}\right) \quad 4
$$

Figure 2 shows the relation between the angle of attack of the airfoil $\alpha$ and azimuth angle $\theta$ at different tip speed ratios $(\lambda)$.

\section{Aerodynamics of unsteady flow}

Since in every revolution of the blade, angle of attack, velocity vectors, and aerodynamic forces vary with time, thus the flow field around the airfoil is unsteady. A lot of experimental, analytical, and numerical works have been done successfully in order to determine the aerodynamic coefficients of stationary flow physics phenomena. Therefore, simulations and predictions of this type of flow have a high degree of accuracy, in contrast to the unsteady aerodynamic flow characteristics. Understanding unsteady aerodynamic is essential to obtain exerted forces on moving objects such as blade of wind turbines. Indeed there are a variety of methods to foresee forces in dynamic mode. Because of some reasons most of these methods are inefficient for wind turbines and have particular limitations and difficulties to implement. One of the significant causes is Reynolds number. The order of magnitude of wind turbines Reynolds number is $10^{5}$ and transition appears on the blade. Another reason which makes the numerical simulations difficult is that the angle of attack of the blade may be close to critical angle of attack. Therefore, in this investigation flow field is simulated in a situation that angle of attack is in the range of static stall angle of attack. In other words, the performance of the blade turbine is analyzed in a corresponding velocity to $\lambda>4$.

As is understood from Fig. 2, when bladerotates 360 degrees around the main axis of turbine angle of attack changes cyclically. At tip speed ratios higher than four the variation curve of angle of attack is very similar to sinusoidal curve. In this range of $\lambda$ the angle of attack of blade during rotation is limited. Therefore, the flow around an airfoil that has pitch oscillation could be simulated in a way that its angle of attack is described by equation 4 which is roughly as same as sine curve. 
As noted, analysis of unsteady flow due to complicated physics is difficult. Reduced frequency, amplitude, Mach number, Reynolds number, and type of pitching oscillation are involved parameters and factors in this complexity. Types of pitching oscillation are harmonic pitching motion in which angle of attack varies according to $\alpha=\alpha_{\text {mean }}+\alpha \sin \omega t$ (Fig. 3) and pitching motion is with steady rate in which the relation between airfoil angle of attack and time is given by the function $\alpha=\alpha_{\text {start }}+\omega t$. Looking at Fig. 2 and considering variation of the angle of attack of the vertical axis wind turbine with time; it is clear that the type of oscillation motion is harmonic.

\section{Geometry and Grid Generation}

In this study, the performance of vertical axis wind turbine with straight blade and NACA 0012, NACA 0015, and NACA 0018 cross sections has been taken into consideration. Gambit software was employed to generate mesh and computational domain.Due to the fact that controlling structured grid is easier and has fewer elements in comparison with unstructured grid; a $\mathrm{C}$ shaped domain which was covered by structured grid was created. The radius of semicircular domain is 16 times chord and the distance between the trailing edge of airfoil and outlet boundary is 19 times chord (Fig. 4).

To improve the quality of mesh especially near the surface of airfoil, the whole computational domain was subdivided into sub domains and mesh of each area was individually produced (Fig. 5). For instance, as could be seen in Fig. 5 this meshing enhances orthogonality near the leading edge.

To ensure the quality of grid before studying airfoils in oscillating mode, the flow around airfoils in static mode and at various angles of attacks was simulated. Results were compared with experiments to ascertain whether were similar or not. If there is an acceptable coincidence, the mesh capability for simulation of the flow around airfoils is verified.

\section{Boundary Conditions}

At the inlet, including the top and bottom boarders of the domain, velocity inlet condition was adopted. The outlet boundary was treated as a pressure outlet. Relative static pressure is set at the outlet boundary. No slip wall condition was employed for the airfoil surface. The free stream temperature and pressure were 300 Kelvin and 1 atmosphere respectively.

\section{Simulation of Flow around Static Airfoil}

In this section two-dimensional flow over NACA 0012 airfoil at three angles of attack is taken into consideration. In previous years a number of tests have been conducted to simulate the flow field of this airfoil and calculate aerodynamic coefficients yet most of experiments have been accomplished at flow regimes with high Reynolds number, of the order of $10^{6}[15]$.

Knowing that vertical axis wind turbines operate at low Reynolds number (magnitude order of $10^{5}$ ) and with lack of reliable data; Sandia National Laboratories evaluated the performance of several NACA four digits airfoils at various Reynolds numbers for use in vertical axis wind turbines and published results in 1981[16]. Therefore, in this study the flow around NACA 0012 airfoils was assessed at different angles of attack and Reynolds number $3.6 \times 10^{6}$ and results were validated with reported data by Sandia National Laboratories. In the next stage, this validation was done for other studied airfoils to ascertain the numerical method reliability and gird independency. In this discussion, the flow field of each airfoil at various angles of attack was deliberately solved when there was not any observation of variation of aerodynamic coefficients namely, lift and drag forces acting on the airfoil.

\section{Grid Independency}

In order to achieve accurate results, the solution must be independent of grid size. Three meshes were created around NACA 0012 airfoil, coarse with 71000, medium with 142000, and fine with 21300 elements. The flow was solved at three angles 3, 6, and 10 degrees and results are presented in Table 1. It is observed that the results of medium and fine meshes are identical. Thus solution is independent of grid and the medium grid was selected.

\section{Turbulence Model}

To find out an appropriate turbulence model, the flow around airfoil at different angles of attack and Reynolds number $3.6 \times 10^{6}$ were solved by adopting Spalart-Allmaras, K- $\varepsilon$, SST (shear stress transport) K- $\omega$, and RSM (Reynolds stress model). Graph of results which shows lift coefficient and drag coefficient against angle of attack, are illustrated in Fig. 6 and Fig. 7 respectively. From Fig. 6, it is clear SST k- $\omega$ has the least error in calculating lift coefficient and prediction of static stall angle. It is also understood from Fig. 7 that SST k- $\omega$ model is a reliable model in prediction of drag coefficient at various angles of attack particularly below the stall 
angle. According to this evaluation; the flow field of other airfoils at Reynolds number $3.6 \times 10^{6}$ was simulated with help of SST k- $\omega$ model.

Because of the importance of the flow behavior near the surface of airfoil, the treatment of analyzing in this region has major role. Likewise in these close regions to the airfoil due to boundary layer, changes of momentum and other variables are more intense thereby, to accomplish more accurate results first grid height was selected in a way that $\mathrm{y}^{+}$was less than 1 all around the airfoil.

\section{Simulation of Unsteady Flow aroundan Airfoil with Sinusoidal Oscillation}

As mentioned previously when blade rotates around the main axis of turbine the blade angle of attack fluctuates in a limited range. These fluctuations are more analogous to sine curve as tip speed ratio exceeds 4 . Thus the flow around the vertical axis turbine blade could be simulated through an oscillating airfoil when angle of attack varies according to equation 4 that is akin to $\alpha=\sin \theta$. The flow around the airfoil with pitching oscillation was evaluated while angle of attack followed function $\alpha=\alpha_{0}+\alpha_{1} \sin \omega t$ and results were compared with experiments.

A number of researchers studied oscillatory airfoils with Reynolds number of $10^{6}$ while for wind turbines it is of order $10^{5}$. In 2004, Lee tested an oscillating NACA 0012 airfoil at Reynolds number $1.3 \times 10^{5}$ with sinusoidal mode $\alpha=\alpha_{0}+\alpha_{1} \sin \omega t$ experimentally [17]. In this study various mean angles of attack $\alpha_{0}$, amplitude of oscillation $\alpha_{1}$, and reduced frequency were studied and hysteresis and aerodynamic forces graphs were plotted.

In present research free stream velocity was selected as 14 meter per second, turbulence intensityand Reynolds number were $0.08 \%$ and $1.3 \times 10^{5}$ respectively. The airfoil oscillated around the quarter chord point of airfoil with an angular frequency of 18.67 radian per second and a reduced frequency of 0.1 . With regard to the range of tip speed ratio the oscillation of airfoil was assessed by functions $\alpha=10 \sin \omega \mathrm{t}$ and $\alpha=15 \sin \omega \mathrm{t}$ and results were compared with experiments.

\section{Numerical Method}

Unsteady two dimensional Navier-Stokes equations were employed for numerical solution. In order to simulate turbulence flow SST k- $\omega$ model which is also appropriate for low Reynolds number flow and its accuracy were confirmed in section 7 was utilized. A time independent UDF (user define function) was provided and compiled through Fluent. Equations including momentum and turbulence were discretized by second order upwind scheme. Furthermore they were coupled by SIMPLE algorithm.

Free stream velocity and turbulence intensity were 14 meter per second and $0.08 \%$ respectively based on experiments. Angular frequency was taken as 18.67 radian per second and pitching magnitude were considered as 10 and 15 degrees. It should be noted that $\mathrm{y}^{+}$around the airfoil was less than 1 . All defaults under relaxations proposed by software were not changed. Convergence criteria of momentum and turbulence were $10^{-}$ 6 and $10^{-4}$ respectively. Time step was chosen according to oscillation frequency. The period of oscillation was calculated with $T=\frac{2 \pi}{\omega}$ and the flow was solved for three different time steps $d t=\frac{T}{100}, \frac{T}{200}$ and $\frac{T}{300}$.

To obtain initial results firstly time independent equations were talked and then and then converged results were used as an initial guess for unsteady problem to accelerate the convergence process. For more accuracy flow equations were solved at three revolutions and the third one was selected as a result for validation. Figure 8 demonstrates variation of lift coefficient of oscillating airfoil versus time which is given by $\alpha=10$ sin $\omega t$ while time step is taken as 0.003 second.

In Fig. 9 the hysteresis graph of lift coefficient of function $\alpha=10 \sin \omega t$ at a variety angles of attack were compared by Lee's work. As it is clear the predicted lift coefficient is quite close with experiment. In addition, the flow of airfoil was evaluated by expression $\alpha=15 \sin \omega t$ ( 15 is the oscillation amplitude) with a reduced frequency of $\mathrm{K}=0.1$. In Fig. 10 hysteresis drag coefficient of this oscillation is plotted together with experimental result.

\section{Simulation of Unsteady Flow over The Blade of Vertical Axis Wind Turbine}

A UDF was written in $\mathrm{C}$ programming language compiled by Fluent to simulate the oscillation of the airfoil of vertical wind turbine at various determined tip speed ratios. Since equation 4 is complex and similar to sine curve in the range of studied tip speed ratio this equation was approximated by an appropriate sinusoidal equation. This approximation was conducted by curve fitting feature of MATLAB. In Fig. 11 this fitted curve for a vertical axis turbine with $\lambda=6$ is displayed.

In this simulation wind velocity $5 \mathrm{~m} / \mathrm{s}$, air density $1.225 \mathrm{~kg} / \mathrm{m}^{3}$ and dynamic viscosity $1.7894 \times 10^{-5}$ were chosen. Based on these values Reynolds number is $3.4 \times 10^{5}$. Design parameters including radius of rotor, chord length, number of blades, and height of blade are presented in Table 2. 
Time step is selected according to oscillation frequency. Due to direct link between oscillation frequency of the airfoil and tip speed ratio at each tip speed ratio a different frequency was considered. In Table 3 these frequencies and tip speed ratios are illustrated.

\section{The Effect of Thickness on Aerodynamic Coefficient of The Blade and Power Coefficient}

As seen from the results of numerical simulations of the flow of vertical axis wind turbine with straight blade associated with various cross sections, the impact of thickness on aerodynamic coefficients of the blade and performance of the turbine were evaluated. Figure 12 displays the changes of lift force acted on three symmetrical airfoils of the vertical axis wind turbine after three revolutions at tip speed ratio of 6 . It is essential to mention that time step was opted 0.004 s. In Fig. 13 drag coefficient hysteresis graph of considered airfoils in range of fluctuated angles of attack at tip speed ratio of 7 is demonstrated. Figure 14 illustrates the power coefficient of the three straight bladed vertical axis wind turbine with studied symmetric airfoils at flow speed of 5 meter per second and based on assumed design parameters, see Table 2.

\section{Conclusion}

In this paper unsteady flow around the blade of vertical axis wind turbine is investigated numerically and the influence of blade thickness on aerodynamic and power coefficients are assessed. Results reveal that increasing thickness leads to reducing lift coefficient and rising drag coefficient at every oscillation. Furthermore power coefficient curves versus various tip speed ratios (Fig. 14) shows that NACA 0012 in the considered tip speed ratio range is more efficient. This conclusion is reasonable with regard to the result of section 11 because the airfoil with the least thickness (NACA 0012) has maximum lift and minimum drag coefficients among examined airfoils.

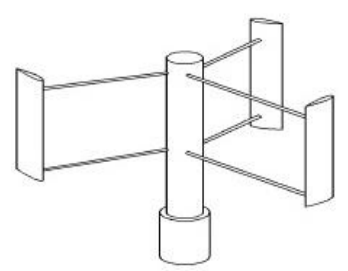

(a)

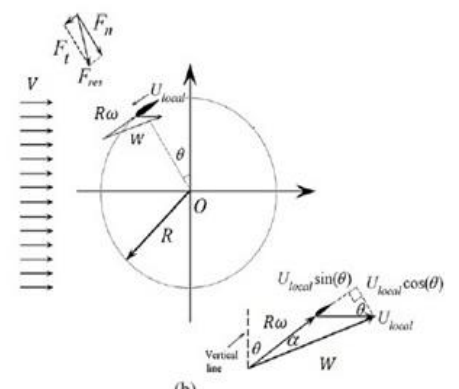

(b)

Fig. 1 (a) Schematic of a three straight bladed wind turbine (b) velocity vector and exerted forces at an arbitrary

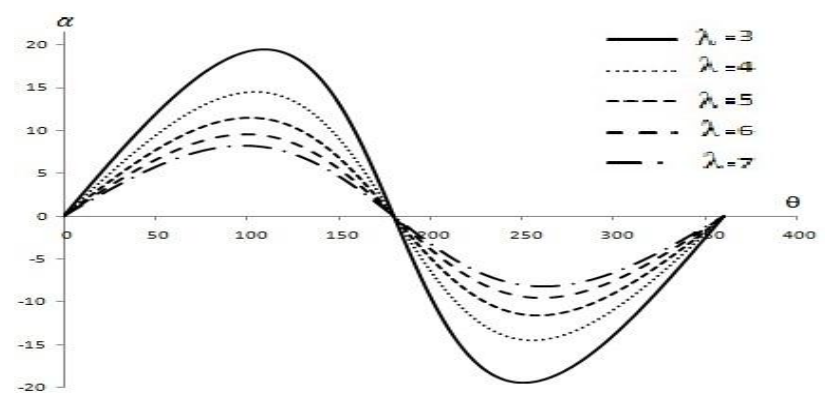

Fig. 2 Variation of angle of attack versus azimuth angle at various



Fig. 3 Schematic of pitching motion of blade 


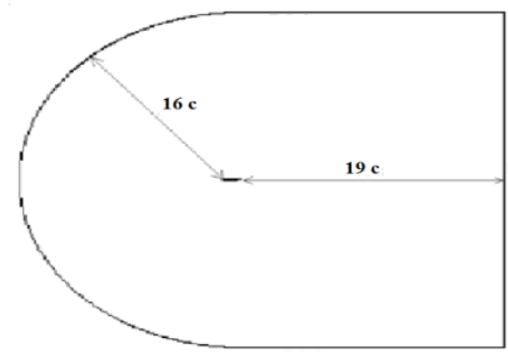

Fig. 4Computational domain

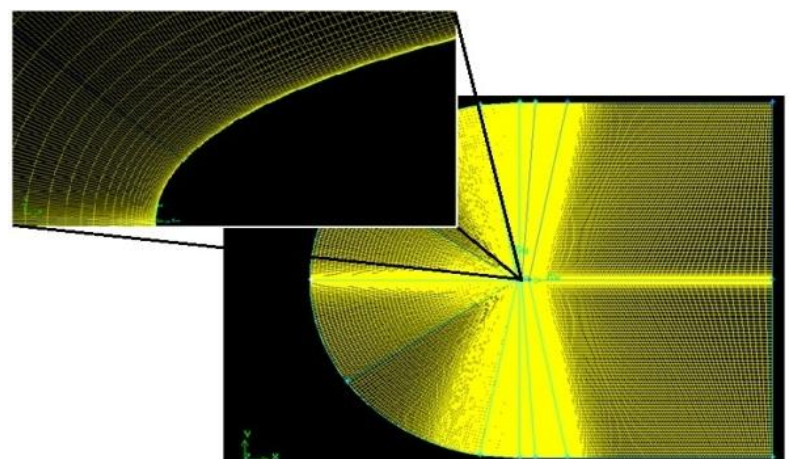

Fig. 5Illustration of mesh around NACA 0012

Table 1Grid study

\begin{tabular}{|r|r|r|r|r|}
\hline angle (degree) & \multicolumn{1}{|c|}{ coefficient } & coarse & medium & fine \\
\hline \multirow{2}{*}{0} & $\mathrm{C}_{\mathrm{l}}$ & 0 & 0 & 0 \\
\cline { 2 - 5 } & $\mathrm{C}_{\mathrm{d}}$ & 0.011 & 0.0095 & 0.0095 \\
\hline \multirow{2}{*}{6} & $\mathrm{C}_{\mathrm{l}}$ & 0.6 & 0.61 & 0.61 \\
\cline { 2 - 5 } & $\mathrm{C}_{\mathrm{d}}$ & 0.014 & 0.013 & 0.013 \\
\hline \multirow{2}{*}{10} & $\mathrm{C}_{\mathrm{l}}$ & 0.94 & 0.96 & 0.96 \\
\cline { 2 - 5 } & $\mathrm{C}_{\mathrm{d}}$ & 0.023 & 0.021 & 0.021 \\
\hline
\end{tabular}

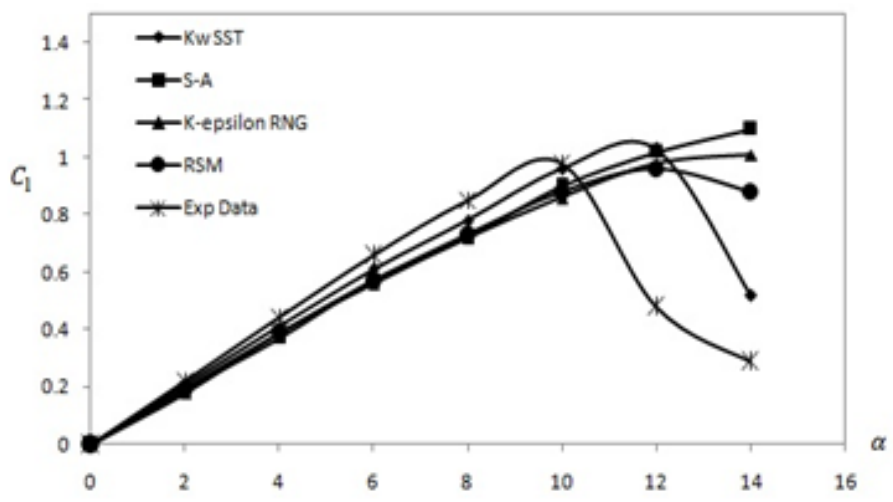

Fig. 6 Comparison of turbulence models (lift coefficient of NACA 0012) and experiment [16]

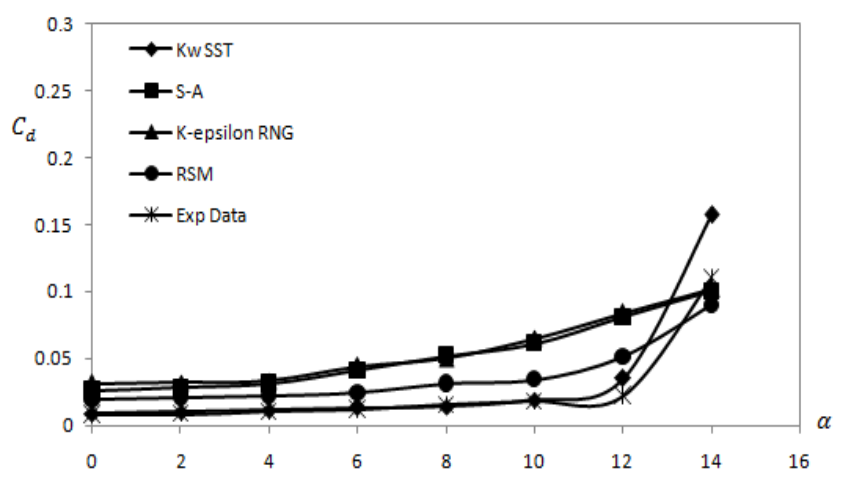

Fig. 7 Comparison of turbulence models (drag coefficient of NACA 0012) and experiment [16] 


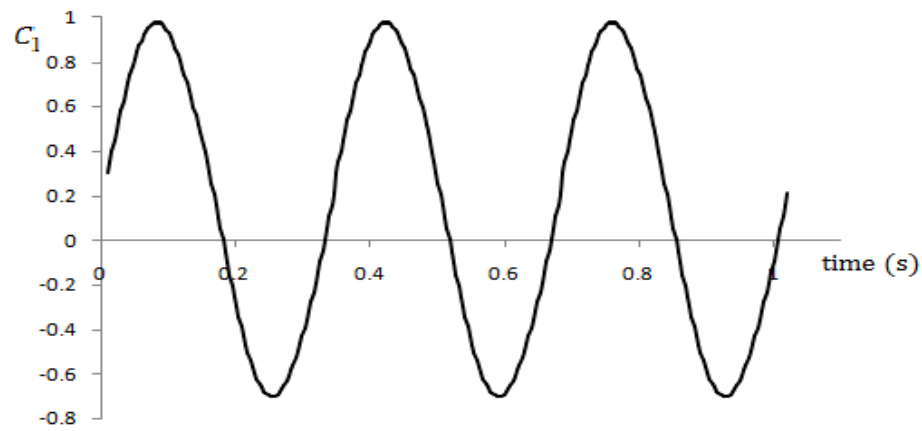

Fig. 8 Changes of lift coefficient with time at the end of third revolution of oscillating NACA 0012, $\alpha=10 \sin \omega t$

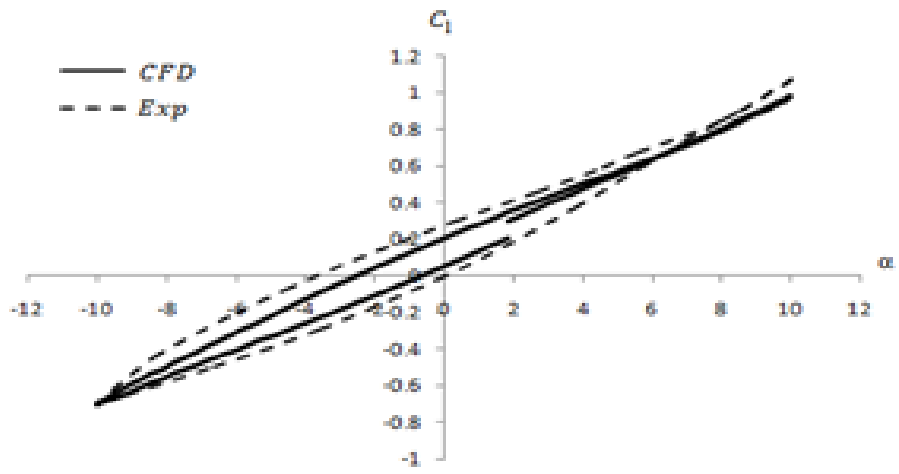

Fig. 9 Comparison of lift coefficient hysteresis with Lee's experiment [17] ( $\alpha=10 \sin \omega t)$



Fig. 10 Comparison of drag coefficient hysteresis with Lee's experiment [17] $(\alpha=10 \sin \omega t)$

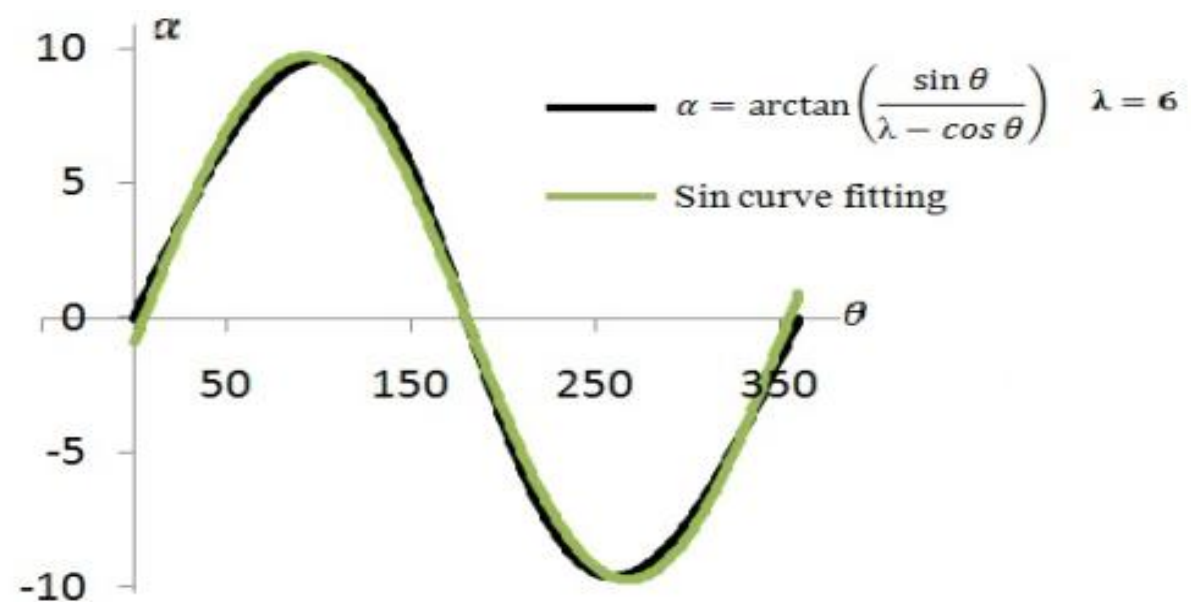

Fig. 11Sinusoidal curve fitting of the blade motion of vertical axis wind turbine at $\lambda=6$ 
Table 2 Design parameters of turbine

\begin{tabular}{|r|r|r|r|}
\hline Rotor radius & Height of blade & Chord & Number of blades \\
\hline 2 & 3 & 0.2 & 3 \\
\hline
\end{tabular}

Table 3 Frequencies corresponding to $\lambda$

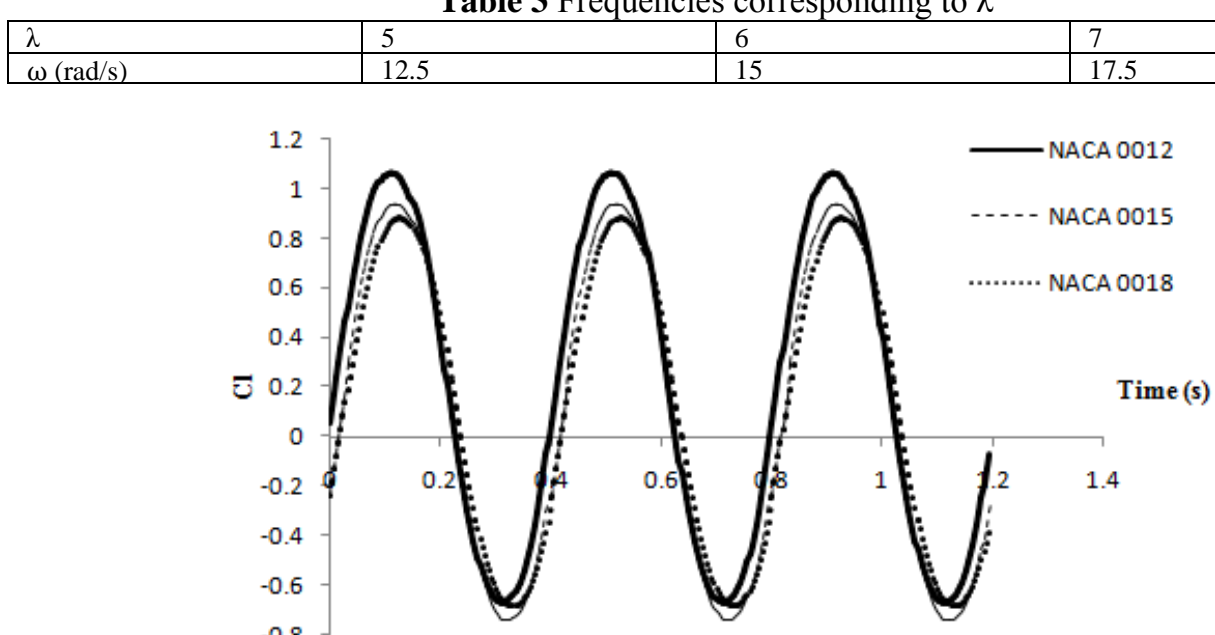

Fig. 12 Lift coefficient of three symmetric airfoils, $\lambda=6$

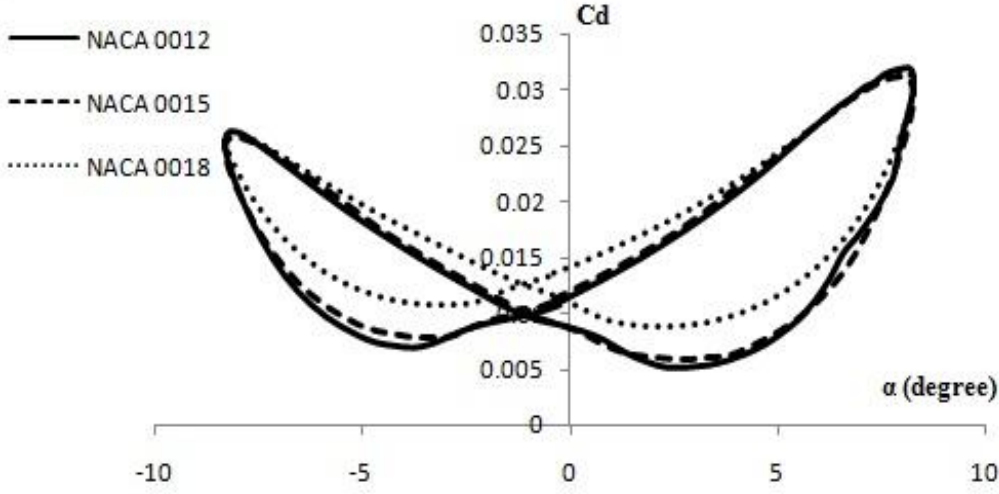

Fig. 13 Drag coefficient of three symmetrical airfoils, $\lambda=7$



Fig. 14 Power coefficient of straight bladed vertical axis wind turbine with three airfoils 


\section{References}

[1] Pope, K.; Rodrigues, V.; Doyle, R.; Tsopelas, A.; Gravelsins, R.; Naterer, G.F.; Tsang, E.: Effects of stator vanes on power coefficients of a zephyr vertical axis wind turbine. Renewable Energy 35(5), 1043-1051 (2010). doi:10.1016/j.renene.2009.10.012

[2] Knight, J.: Urban wind power: Breezing into town. Nature 430(6995), 12-13 (2004).

[3] Mertens, S.: Wind energy in the built environment: concentrator effects of buildings. TU Delft, Delft University of Technology, (2006)

[4] Berg, D.E.: Structural design of the Sandia 34-meter vertical-axis wind turbine. Sandia National Laboratories, (1985)

[5] Van Bussel, G.: A momentum theory for winglets on horizontal axis wind turbine rotors and some comparison with experiments. In: Fourth IEA Symposium on the Aerodynamics of Wind Turbines 1990. Rome, Italy. Proc. edt. K. McAnulty, ETSU-N-118, Harwell, Didcot, UK

[6] Stoddard, F.S.: Discussion Of Momentum Theory For Windmills. (1976)

[7] Hartwanger, D.; Horvat, A.: 3D modeling of a wind turbine using CFD. In: NAFEMS Conference, United Kingdom 2008

[8] Coppens, A.J.; Jones, S.; Davis Engineering, L.; Canada; Energy, M.; Resources, C.: Investigation of alternative materials for use in mid-size vertical axis wind turbine blades : materials assessment. Technology Branch, Energy, Mines and Resources, Ottawa (1989)

[9] Islam, M.; Ting, D.; Fartaj, A.: Design of a Special-purpose Airfoil for Smaller-Capacity Straight-Bladed VAWT. Wind Engineering 31(6), 401-424 (2007). doi:doi:10.1260/030952407784079780

[10] Islam, M.: Analysis of Fixed-Pitch Straight-Bladed VAWT with Asymmetric Airfoils. University of Windsor (2008)

[11] Howell, R.; Qin, N.; Edwards, J.; Durrani, N.: Wind tunnel and numerical study of a small vertical axis wind turbine. Renewable Energy 35(2), 412-422 (2010). doi:10.1016/j.renene.2009.07.025

[12] Wang, S.; Ingham, D.B.; Ma, L.; Pourkashanian, M.; Tao, Z.: Numerical investigations on dynamic stall of low Reynolds number flow around oscillating airfoils. Computers \& Fluids 39(9), 1529-1541 (2010). doi:10.1016/j.compfluid.2010.05.004

[13] Beri, H.; Yao, Y.: Effect of camber airfoil on self starting of vertical axis wind turbine. Journal of environmental Science and Technology 4(3), 302-312 (2011).

[14] Castelli, M.R.; De Betta, S.; Benini, E.: Effect of blade number on a straight-bladed vertical-axis Darreius wind turbine. World Academy of Science, Engineering and Technology 61, 305-3011 (2012).

[15] Ladson, C.L.; United, S.: Effects of independent variation of Mach and Reynolds numbers on the low-speed aerodynamic characteristics of the NACA 0012 airfoil section. NASA technical memorandum ;4074, vol. 94 p. National Aeronautics and Space Administration, Scientific and Technical Information Division, Washington, D.C. (1988)

[16] Sheldahl, R.E.; Klimas, P.C.: Aerodynamic characteristics of seven symmetrical airfoil sections through 180-degree angle of attack for use in aerodynamic analysis of vertical axis wind turbines. In. Sandia National Labs., Albuquerque, NM (USA), (1981)

[17] Lee, T.; Gerontakos, P.: Investigation of flow over an oscillating airfoil. Journal of Fluid Mechanics 512, 313-341 (2004). 